\title{
Comparison the effect on cerebral oxygenation by sevoflurane-remifentanil or propofol-remifentanil anesthesia in patients undergoing carotid endarterectomy
}

\section{Soengtae Jeong, Sung-Su Chung, Jeongmin Kim, En-A Jang, Hyung-Jeong Lee}

Department of Anesthesiology and Pain Medicine, Medical School, Chonnam National University, Gwangju, Korea

\section{INTRODUCTION}

$>$ Carotid endarterectomy (CEA)

$\checkmark$ Surgical treatment of carotid artery stenosis

$\checkmark$ Cerebral hypoperfusion after carotid artery clamping

$\checkmark$ Cerebral hyperperfusion syndrome after declamping (rare)

$>$ Regional cerebral oxygen saturation $\left(\mathrm{SrO}_{2}\right)$

$\checkmark$ Balance of oxygen supply and demand of brain tissue

$\checkmark$ Near-infrared spectroscopy (NIRS)

- non-invasive tool to monitor the balance of cerebral oxygenation continuously

$>$ Sevoflurane vs. Propofol

$\checkmark$ Both agents decrease the CMRO2 in a similar manner

$\checkmark \mathrm{CMRO}_{2} \downarrow \rightarrow \mathrm{CBF} \downarrow$

$\checkmark$ Sevoflurane

$\checkmark$ Vasodilation effect $\rightarrow \mathrm{CBF} \uparrow$

$\checkmark$ CBF $>$ cerebral oxygen demand (luxury perfusion)

$\checkmark$ Propofol

$\checkmark$ Vasoconstriction effect $\rightarrow \mathrm{CBF} \downarrow$

$\checkmark \mathrm{CMRO}_{2} \downarrow<\mathrm{CBF} \downarrow \downarrow(\mathrm{CBF} / \mathrm{CMRO} 2$ ratio $\downarrow)$

$\checkmark$ The aim of study

$\checkmark$ compare the effects of sevoflurane and propofol to $\mathrm{SrO}_{2}$ in the patients scheduled CEA to treat the defect of cerebral blood flow.

\section{MATERIALS \& METHODS}

Patients \& experimental group

$\checkmark \quad 74$ patients aged 19-80 year, scheduled to undergo carotid endarterectomy under general anesthesia

$\checkmark$ Randomly allocated to Sevoflurane or Propofol group

$\checkmark$ Exclusion criteria

- ASA physical status $>4$,

- Preoperative $\mathrm{SpO}_{2}<97 \%$,

- Both carotid arteries stenosis

- Co-existing other neurological disease

$>$ Anesthesia

$\checkmark$ Premedication: triazolam $0.125 \mathrm{mg}$ p.o

$\checkmark$ Remifentanil 3 ng/ml using target controlled infuser (TCI)

$\checkmark$ Sevoflurane or propofol TCI: BIS $40 \sim 50$

$\checkmark$ Rocuronium $0.6 \mathrm{mg} / \mathrm{kg}+$ continuous infusion $(8 \mu \mathrm{g} / \mathrm{kg} / \mathrm{min})$

$\checkmark$ Mechanical ventilation: PaCO2 $35 \sim 40 \mathrm{mmHg}$

$\checkmark$ MAP $<55$ mmHg or $25 \%$ of baseline, SBP $<90 \mathrm{mmHg}$

- Phenylephrine $100 \mu \mathrm{g}$ iv

- Dopamine infusion: if hypotension sustatined

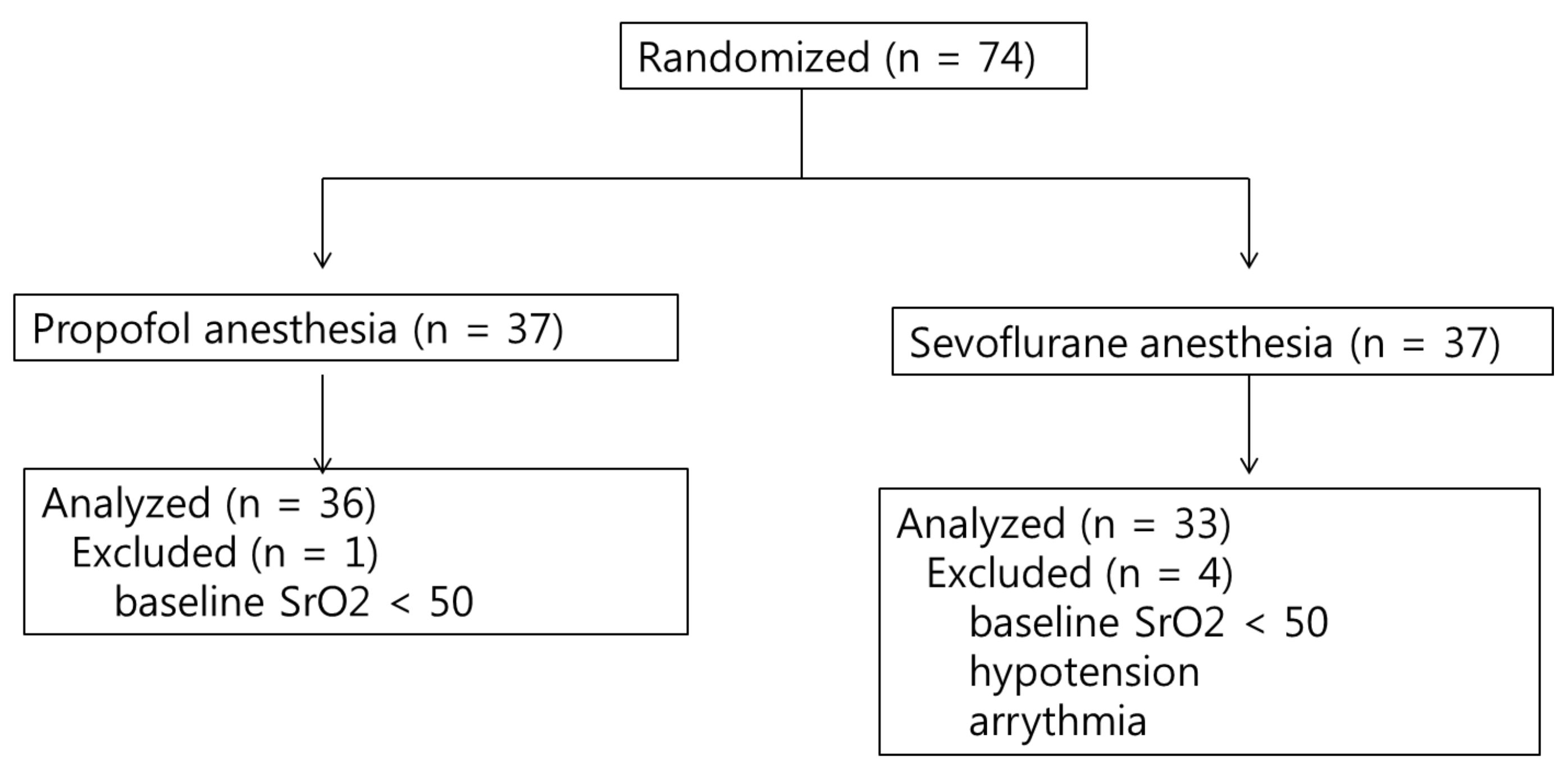

\section{RESULTS}

Table 1. Demographic and Intraoperative Variables

\begin{tabular}{|c|c|c|c|c|}
\hline & Sevoflurane $(\mathrm{n}=36)$ & \multicolumn{2}{|c|}{ Propofol $(n=33)$} & P value \\
\hline Sex (Male/Female) & $31 / 5$ & \multicolumn{2}{|c|}{$27 / 6$} & 0.562 \\
\hline Age & $69 \pm 8$ & \multicolumn{2}{|c|}{$67 \pm 7$} & 0.212 \\
\hline Height $(\mathrm{cm})$ & $163 \pm 7$ & \multicolumn{2}{|c|}{$164 \pm 7$} & 0.322 \\
\hline Weight(kg) & $64 \pm 10$ & \multicolumn{2}{|c|}{$66 \pm 10$} & 0.513 \\
\hline Hemoglobin $(\mathrm{g} / \mathrm{dl})$ & $12 \pm 1$ & \multicolumn{2}{|c|}{$13 \pm 1$} & 0.273 \\
\hline \multicolumn{5}{|l|}{ Underlying diseases } \\
\hline Hypertension & 30 & \multicolumn{2}{|c|}{24} & 0.413 \\
\hline DM & 13 & \multicolumn{2}{|c|}{15} & 0.389 \\
\hline Total anesthesia time & $181 \pm 29$ & \multicolumn{2}{|c|}{$174 \pm 26$} & 0.914 \\
\hline Total operation time & $161 \pm 19$ & \multicolumn{2}{|c|}{$158 \pm 17$} & 0.806 \\
\hline Artery clamping time & $39 \pm 8$ & \multicolumn{2}{|c|}{$38 \pm 10$} & 0.401 \\
\hline \multicolumn{5}{|l|}{ Fluid administered (ml) } \\
\hline Crystalloid & $1412 \pm 377$ & \multicolumn{2}{|c|}{$1618 \pm 441$} & 0.069 \\
\hline Colloid & $338 \pm 218$ & \multicolumn{2}{|c|}{$256 \pm 237$} & 0.201 \\
\hline Bleeding (ml) & $90 \pm 42$ & \multicolumn{2}{|c|}{$116 \pm 72$} & 0.159 \\
\hline Urine $(\mathrm{ml})$ & $577 \pm 279$ & \multicolumn{2}{|c|}{$762 \pm 400$} & 0.109 \\
\hline \multicolumn{5}{|c|}{ Values are mean \pm SD or numbers. } \\
\hline \multicolumn{5}{|c|}{ Table 3. $\mathrm{SrO}_{2}$ Values in before arterial clamping } \\
\hline & & $\begin{array}{c}\text { Sevoflurane } \\
(\mathrm{n}=36)\end{array}$ & $\begin{array}{c}\text { Propofol } \\
(\mathrm{n}=33)\end{array}$ & P-value \\
\hline \multicolumn{5}{|l|}{$\overline{\text { Ipsilateral }}$} \\
\hline Mean $\mathrm{SrO}_{2}(\%)$ & & $63.9 \pm 6.6$ & $65.3 \pm 6.4$ & 0.452 \\
\hline Minimum $\mathrm{SrO}_{2}(\%)$ & & $61.8 \pm 6.6$ & $63.1 \pm 7.1$ & 0.407 \\
\hline $\begin{array}{l}\text { Mean value of relative change } \\
\text { compare to baseline }(\%)\end{array}$ & \multicolumn{3}{|c|}{ Mean value of relative changes in $\mathrm{SrO}_{2}$} & 0.237 \\
\hline \multicolumn{5}{|c|}{ Relative maximum decrease in $\mathrm{SrO}_{2}(\%)$} \\
\hline compare to baseline & & $3.3 \pm 8.8$ & $4.8 \pm 9.7$ & 0.504 \\
\hline compare to post-induction & & $9.9 \pm 6.2$ & $8.1 \pm 6.2$ & 0.250 \\
\hline \multicolumn{5}{|l|}{ Contralateral } \\
\hline Mean $\mathrm{SrO}_{2}(\%)$ & & $67.1 \pm 7.5$ & $67.3 \pm 6.9$ & 0.452 \\
\hline Minimum $\mathrm{SrO}_{2}(\%)$ & & $66.3 \pm 8.0$ & $66.3 \pm 7.1$ & 0.992 \\
\hline Mean value of relative changes in $\mathrm{SrO}_{2}$ & & $9.1 \pm 20$ & $-1.9 \pm 22$ & 0.033 \\
\hline \multicolumn{5}{|c|}{ Relative maximum decrease in $\mathrm{SrO}_{2}(\%)$} \\
\hline compare to baseline & & $-3.9 \pm 10.1$ & $0.7 \pm 10.5$ & 0.067 \\
\hline compare to post-induction & & $4.4 \pm 3.7$ & $6.6 \pm 5.1$ & 0.038 \\
\hline
\end{tabular}
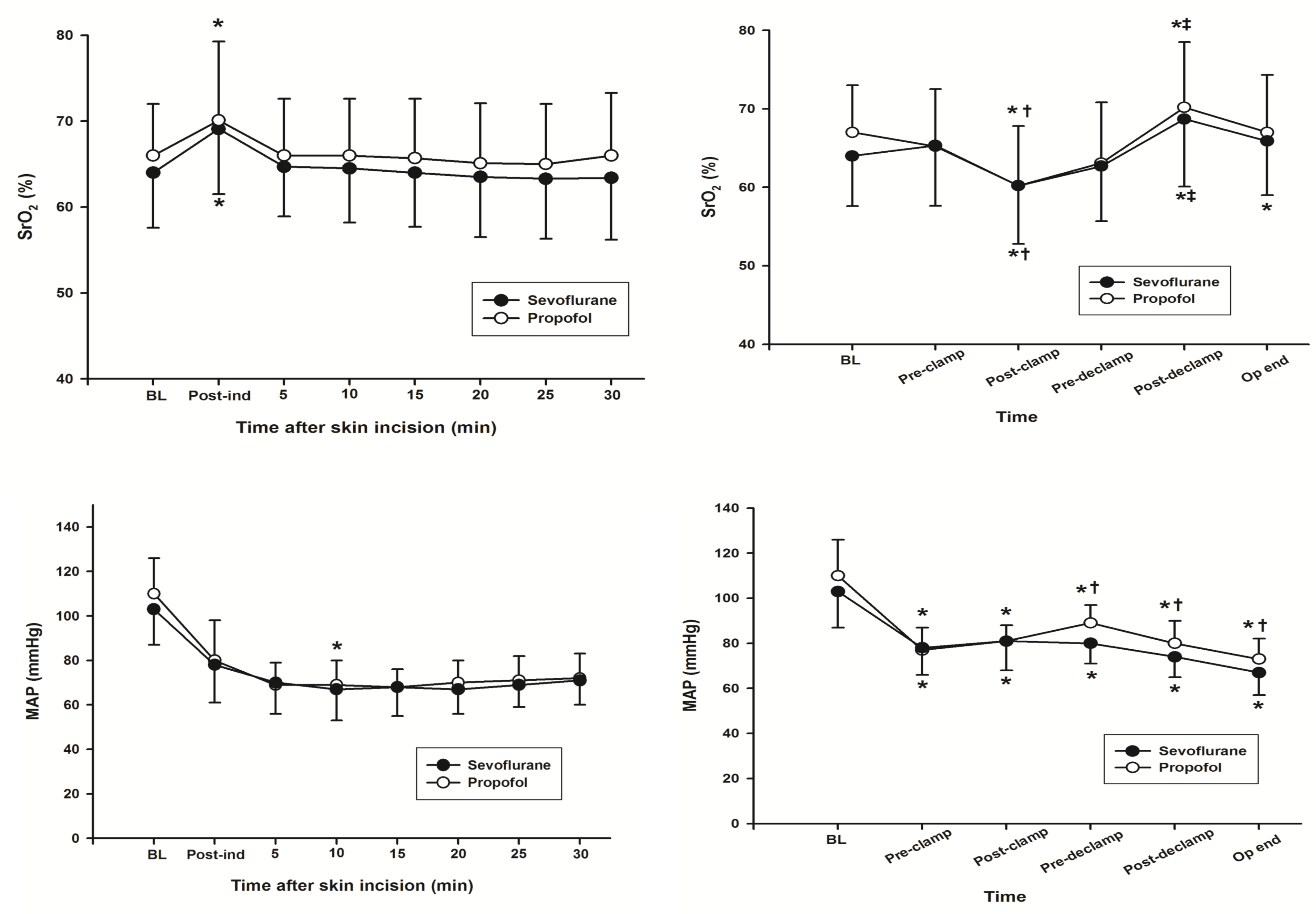

\section{CONCLUSION}

Propofol-remifentanil anesthesia was comparable with sevofluraneremifentanil anesthesia undergoing carotid endarterectomy in an aspect of preserving the $\mathrm{SrO}$, with less decrease in mean arterial blood pressure. 